\title{
Acute Psychosis in a Child with Severe Dengue
}

\author{
Bhatnagar $\mathbf{R}^{1}$, Prasad $\mathbf{P}^{2}$
}

\begin{abstract}
Dengue fever is emerging as an important cause of acute febrile illness with neuropsychiatric symptoms in adult population especially in endemic areas. Numerous case reports and review articles have already been published in past emphasizing on neurological manifestations in dengue but sufficient data on psychiatric symptoms in paediatric age group is still lacking. Acute psychosis in recovery phase of severe dengue is an uncommon phenomenon, thus rarely reported.
\end{abstract}

Key words: Dengue fever, acute psychosis

\section{Introduction}

$\mathrm{D}$ engue is the most rapidly spreading mosquito-borne viral disease in the world. In the last 50 years, incidence has increased 30 fold with increasing geographic expansion to new countries and in the present decade, from urban to rural settings. It is due to the resurgence of vector Aedes ageypti, overcrowding and increasing travel ${ }^{1}$. It often constitute a public health emergency of national and international concern with implications for health security due to disruption and rapid epidemic spread beyond national borders ${ }^{2}$.

There is substantial evidence in literature linked to neurological complications in severe dengue of which encephalitis ${ }^{3}$, encephalopathy, mania, depression have been reported in past ${ }^{4,5,6}$. Acute psychosis as post-infectious sequelae in severe dengue is rare. The rarity of such an event has prompted us to report this case.

\section{The Case}

A 14 year old male, admitted with complaints of high grade fever associated with chills and occasional vomiting for three days. There was no history of headache, seizures, ear discharge, and dysuria. On general examination, patient was conscious with BP 100/60 $\mathrm{mmHg}$, with heart rate $110 / \mathrm{min}$ and signs of adequate peripheral perfusion. Systemic examination revealed hepatosplenomegaly with liver $2.5 \mathrm{~cm}$ and spleen $1.5 \mathrm{~cm}$ palpable below right and left subcostal margin respectively. Investigations sent on the day of admission revealed positive NS1 antigen, with platelet count of $65,000 /$ cumm and raised liver enzyme levels; AST $1116 \mathrm{IU} / \mathrm{ml}$ and ALT $628 \mathrm{IU} / \mathrm{ml}$. Serum electrolytes, renal function tests and coagulation profile were normal. Peripheral blood smear was negative for malarial parasite.
${ }^{1}$ Dr Ruchika Bhatnagar, MBBS. MD. Assistant Professor. ${ }^{2}$ Dr. Premlochan Prasad, MBBS. MD. Professor and Head. Both from the Department of Paediatrics, Shri Ram Murti Smark Institute of Medical Sciences (SRMSIMS), Bareilly, India

\section{Address for correspondence \\ Dr. Ruchika Bhatnagar \\ E-mail: ruchu.bhatnagar@gmail.com}

\section{How to cite}

Bhatnagar R, Prasad P. Acute Psychosis in a Child with Severe Dengue. J Nepal Paediatr Soc 2017;37(3):273-275.

doi:http://dx.doi.org/10.3126/jnps.v37i3.16481

This work is licensed under a Creative Commons Attribution 3.0 License. 
He was diagnosed and managed as a case of dengue fever with warning signs. On the third day of admission, patient was afebrile but developed abdominal pain and clinical features suggestive of compensated shock with BP 94/60 mmHg, cold peripheries, low volume pulse and prolonged capillary refill time. He received fluid resuscitation and supportive treatment based on WHO Guidelines for management of severe dengue. During evening hours, he had 2-3 episodes of melena following which coagulation profile was repeated which showed prolonged PT and aPTT values of 22.3 seconds (s) and 57.8 s respectively, platelet count was 75,000 /cumm. Whole blood transfusion was given and fluid management was continued. Next morning he developed profuse bleed per rectum for which whole blood was again transfused, later on he developed respiratory distress which required ventilator support. Chest X-ray and ABG were consistent with findings of Acute Respiratory Distress Syndrome (ARDS). After two days of ventilatory support, respiratory distress resolved and he was extubated. On the sixth day of admission, dengue serology was repeated which showed seroconversion and raised IgG titres indicating secondary dengue infection. Serial monitoring of platelet count, PT, aPTT values showed improvement along with decrease in liver enzymes levels to normal values. The next day, he developed aggressive behaviour, irritability, irrelevant talking for which he was reassessed neurologically and did not reveal abnormality. Fundus, CSF examination and metabolic parameters were normal. MRI Brain was done to rule out underlying organic cause for neuropsychiatric symptoms but showed no abnormality. Personal and family history was non-contributory. Therefore psychiatric evaluation was considered and the mental status examination revealed features of acute psychosis with delirium. He was treated with tab haloperidol $0.25 \mathrm{mg}$ twice a day for three days. Gradually the psychiatric symptoms abated with complete recovery in a fortnight following which he was discharged. No recurrence of psychiatric symptoms observed after cessation of drug therapy.

\section{Discussion}

The incidence of dengue has grown dramatically around the world in last few decades. One recent estimate indicates 390 million dengue infections per year of which 96 million manifest clinically ${ }^{7}$. An estimated 500,000 people with severe dengue require hospitalization each year, a large proportion of which are children, and about $2.5 \%$ of those affected die.

Neurological manifestations are commonly known to occur in dengue which include mental irritability, altered sensorium, headache, dizziness, convulsions attributable to prolonged shock, metabolic acidosis, severe disseminated intravascular coagulopathy, hepatic and renal dysfunction ${ }^{8}$. Meticulous literature search strategy was done using PUBMED, MEDLINE, EMBASE database to review the incidence and outcome of psychiatric illness in dengue in the paediatric population but the data obtained was insufficient.

The incidence of dengue psychosis is primarily limited to adults in the 18-65 years group as revealed in previous studies. Jhanjee et. al. ${ }^{9}$ conducted a study to assess the psychiatric symptomatology in dengue fever and its progression over the course of illness. They observed that out of 953 confirmed dengue cases, 110 were found to have identifiable psychiatric symptoms, nearly all cases (90.3\%) exhibited thanatophobia (fear of own death). Over $80 \%$ of the cases exhibited clinically significant anxiety and associated symptoms. Around one fifth of the subjects (mostly females) had panic attacks and only less than $15 \%$ of them needed short course of anxiolytics. All the observed psychiatric symptoms decreased both in frequency and severity during recovery phase. In our case, psychosis was evident during recovery phase as contrary to previous reports where it was manifested during the acute phase of illness. Most of the patients with psychotic features required reassurance and support except few ${ }^{10}$ where olanzapine, lorazepam were given for few weeks to resolve the symptoms. Among the various psychiatric symptoms complicating dengue illness, mania is most frequently reported ${ }^{11}$. Other psychiatric symptoms reported include auditory hallucinations, delusions in a 61 year old man suffering from dengue fever which necessitated treatment with oral valproate and quietapine $^{12}$.

\section{Conclusion}

This case elucidates the fact that acute psychotic features manifesting as delirium can occur in older children as late manifestation of severe dengue which may coincide with recovery phase. Dengue illness should be considered as one of the differentials in children presenting with fever, neuropsychiatric symptoms, and who have been residing in endemic area or during epidemic outbreaks. 


\section{References}

1. Solomon T, Dung NM, Vaughn DW, Kneen R, Thao LT, Raengsakulrach $B$, et al. Neurological manifestations of dengue infection. Lancet 2000; 355:1053-9. DOI: 10.1016/S0140-6736(00)02036-5

2. WHO. Dengue and dengue haemorrhagic fever. Factsheet No 117, revised May 2008. Geneva, World Health Organization, 2008 (Accessed from: http:// www.who.int/mediacentre/factsheets/fs117/en/).

3. Garcia-Rivera EJ, Rigau-Perez JG. Encephalitis and dengue. Lancet 2002;360:261. DOI: 10.1016/S01406736(02)09481-3

4. Blum JA, Pfeifer S, Hatz CF. Psychiatric manifestations as the leading symptom in an expatriate with dengue fever. Infection 2010;38(4):341. DOI: 10.1007/s15010010-0029-9. Epub 2010 Jun 22.

5. Palma-da Cunha-Matta A, Soares-Moreno SA, Cardoso-de Almeida A, Aquilera-de Freitas V, CarodArtal FJ. Neurological complications arising from dengue viral infection. Rev Neurol 2004;39:233-37.
6. Jhanjee A, Bhatia MS, Srivastava S. Mania in Dengue Fever. Ind Psychiatr J 2011;20(1):56-7. DOI: 10.4103/0975-2870.202111

7. Bhatt S, Gething PW, Brady P, Messina JP. The global distribution and burden of dengue. Nature 2015;496:504-7. DOI: 10.1038/nature12060. Epub 2013 Apr 7.

8. Morys JM, Jezewska M, Neuropsychiatric manifestations of some tropical diseases. Int Marit Health 2015;66:30-35. DOI: 10.5603/IMH.2015.0009

9. Jhanjee A, Bhatia MS A Study of Psychiatric Symptomatology in Dengue Patients. Delhi Psychiatr J 2013;16(1):21-39

10. Kar S. Post Dengue Psychosis. Indian J Biol Psychiatr 2013;1(1):58-9. DOI: 10.4103/0975-2870.202104

11. Mendhekar DN, Aggarwal P, Aggarwal A. Classical mania associated with dengue infection. Indian $\mathrm{J}$ Med Sci 2006; 60 : 115-16

12. Tripathi SM ,Mishra N. Late Onset Mania in Dengue Fever. Immunol Infect Dis 2014;2(1):1-3. DOI: 10.13189/iid.2014.020101. 